\title{
Liouville's theorem in the radially symmetric case
}

\author{
Richard Froese \\ Department of Mathematics, \\ University of British Columbia, \\ Vancouver, British Columbia, Canada, V6T $1 Z$ \\ rfroese@math.ubc.ca
}

August 8, 2003

\section{Abstract}

We present a very short proof of Liouville's theorem for solutions to a non-uniformly elliptic radially symmetric equation. The proof uses the Ricatti equation satisfied by the Dirichlet to Neumann map.

Mathematics subject classification numbers: primary: 35B05 secondary: 34A30

\section{Introduction}

The classical version of Liouville's theorem asserts that if a harmonic function defined on all of Euclidean space is bounded, it must be constant. This fundamental result has been generalized in many directions, and its study for manifolds is a large field of research. Several years ago, in connection with their work on the De Giorgi conjecture, Ghoussoub and Gui [GG] raised the question of whether Liouville's theorem holds in Euclidean space for solutions of non uniformly elliptic equations of the form

$$
\nabla \cdot \sigma^{2} \nabla \varphi=0
$$

in place of harmonic functions. Here $\sigma(x)^{2}$ is bounded and positive, but need not be bounded away from zero. It was known from the work of Berestycki, Caffarelli and Nirenberg [BCN] that in two dimensions this version of Liouville's theorem holds. However Ghoussoub and Gui [GG] showed that that in dimensions 7 and higher it does not. The remaining cases, dimensions 3 through 6 were settled, also in the negative, by Barlow [B].

The situation is quite different if $\sigma$ is assumed to be radially symmetric. In this case Liouville's theorem does hold in any dimension. This is known from work of Losev [L] (see also [LM], which contains further references). Of course, the radially symmetric case is much simpler, since separation of variables 
reduces the problem to question about ODE's. Specifically, any solution $\varphi$ is a linear combination of solutions of the form $\varphi(x)=u(|x|) Y(x /|x|)$, where $Y$ is an eigenfunction of the spherical Laplacian with eigenvalue $\mu^{2}$, and $u$ satisfies the ODE (1) below.

This paper is just a small remark about this simple case. We show that the Ricatti equation for the Dirichlet to Neumann map leads to a very short proof. Of course, in our setting the Dirichlet to Neuman map is just a function, namely the function $f$ defined by (3) below. But it is interesting to note that the Dirichlet to Neumann map satisfies an operator version of the same Ricatti equation (4) that $f$ satisfies, even when $\sigma$ is not radially symmetric. Perhaps this can be used to give a proof of Liouville's theorem for perturbations of radially symmetric $\sigma$. However, we did not see a simple way of doing this.

\section{Growth of solutions}

Theorem 1 Suppose that $u$ solves the radial Laplace equation

$$
\left(\sigma^{2}(r) u^{\prime}(r)\right)^{\prime}+\frac{(n-1) \sigma^{2}(r)}{r} u^{\prime}(r)-\frac{\mu^{2} \sigma^{2}(r)}{r^{2}} u(r)=0
$$

for $r \in(0, \infty)$. Assume $n \geq 3$ and $\sigma^{2}(r) \in C^{1}([0, \infty))$ with $0<\sigma^{2}(r) \leq 1$. Define $\beta(\mu)$ to be

$$
\beta(\mu)=-\frac{n-2}{2}+\sqrt{\left(\frac{n-2}{2}\right)^{2}+\mu^{2}}
$$

If $u$ is bounded by

$$
|u(r)| \leq C\left(1+r^{\beta}\right)
$$

then $\beta \geq \beta(\mu)$.

Remark: This theorem gives a minimal growth rate, depending on $\mu$, for a solution $u$ to the radial Laplace equation. It implies Liouville's theorem, since a bounded solution $(\beta=0)$ is only possible when $\beta(\mu)=0$. In this case $\mu=0$, and $u(r)=C$ is the only solution bounded at the origin.

Remark: When $\sigma(r)=1$ the solution bounded at the origin is $u(r)=r^{\beta(\mu)}$. Thus the value of $\beta(\mu)$ is optimal.

Proof: Suppose that $u$ is a solution satisfying (2). We must show that $\beta \geq \beta(\mu)$.

Define

$$
f(r)=\frac{r^{n-1} \sigma^{2}(r) u^{\prime}(r)}{u(r)}
$$


Then $f$ satisfies the Ricatti type equation

$$
f^{\prime}(r)=r^{n-3} \mu^{2} \sigma^{2}(r)-\frac{1}{r^{n-1} \sigma^{2}(r)} f^{2}(r)
$$

We begin by showing that $f$ is well defined and positive. This follows from the ODE version of Calderon's identity, namely,

$$
\sigma^{2}(r) u^{\prime}(r) u(r) r^{n-1}=\int_{0}^{r} \sigma^{2}(s)\left(u^{\prime}(s)^{2}+\frac{\mu^{2}}{s^{2}} u^{2}(s)\right) s^{n-1} d s>0
$$

To prove this we first integrate by parts to obtain

$$
\begin{aligned}
0 & <\int_{a}^{r} \sigma^{2}(s)\left(u^{\prime}(s)^{2}+\frac{\mu^{2}}{s^{2}} u^{2}(s)\right) s^{n-1} d s \\
& =\left.\sigma^{2}(s) u^{\prime}(s) u(s) s^{n-1}\right|_{a} ^{r}-\int_{a}^{r} u(s)\left\{\frac{d}{d s}\left(\sigma^{2} u^{\prime} s^{n-1}\right)-\frac{\mu^{2}}{s^{2}} u^{2}(s) s^{n-1}\right\} d s \\
& =\left.\sigma^{2}(s) u^{\prime}(s) u(s) s^{n-1}\right|_{a} ^{r}
\end{aligned}
$$

Then (5) follows from

$$
\lim _{a \rightarrow 0} \sigma^{2}(a) u^{\prime}(a) u(a) a^{n-1}=0
$$

To see this, notice that the equation implies that $\left(\sigma^{2} r^{n-1} u^{\prime}\right)^{\prime}=\mu^{2} \sigma^{2} r^{n-3} u$, so that the bounds on $\sigma^{2}$ and $u$ imply that near zero,

$$
0 \leq\left(\sigma^{2} r^{n-1} u^{\prime}\right)^{\prime} \leq C r^{n-3}
$$

Integrating from 0 to $a$ and letting $a$ tend to zero gives (7) and thus (5). This shows that neither $u$ nor $u^{\prime}$ can vanish, and that $f$ is well defined, does not blow up, and is positive. Since $u$ cannot change sign, so we may as well assume that $u>0$.

The idea of the proof is to estimate the quantity

$$
Q(r)=\int_{1}^{r} u^{\prime}(x) / u(x)+\epsilon f^{\prime}(x) / f(x) d x
$$

from above and below for large $r$.

We begin with the upper bound. Performing the integral in (8) yields

$$
Q(r)=\ln (u(r))+\epsilon \ln (f(r))+C
$$

Here $C$ denotes a generic constant that may depend on $\mu$ but not $r$. Dropping the negative second term in (4) and recalling that $\sigma^{2}(r) \leq 1$ yields

$$
f^{\prime}(r) \leq r^{n-3} \mu^{2} \sigma^{2}(r) \leq r^{n-3} \mu^{2}
$$


Integrating this yields

$$
f(r) \leq \frac{\mu^{2}}{n-2} r^{n-2}+C
$$

This estimate together with our growth assumptions on $u$ imply

$$
Q(r) \leq \beta \ln (r)+\epsilon(n-2) \ln (r)+C
$$

Now we turn to the lower bound. Using the expression given by (4) for $f^{\prime}$ we obtain

$$
\begin{aligned}
u^{\prime}(x) / u(x)+\epsilon f^{\prime}(x) / f(x) & =\frac{f(r)}{r^{n-1} \sigma^{2}(r)}+\epsilon\left(\frac{r^{n-3} \mu^{2} \sigma^{2}(r)}{f(r)}-\frac{f}{r^{n-1} \sigma^{2}(r)}\right) \\
& \geq(1-\epsilon) \frac{f(r)}{r^{n-1} \sigma^{2}(r)}+\epsilon \frac{r^{n-3} \mu^{2} \sigma^{2}(r)}{f(r)}
\end{aligned}
$$

Now assume that $0 \leq \epsilon \leq 1$. Since for positive numbers $a, b$ and $c$ we have

$$
a c+b / c \geq 2 \sqrt{a b}
$$

we find

$$
u^{\prime}(x) / u(x)+\epsilon f^{\prime}(x) / f(x) \geq 2 \mu \sqrt{\epsilon(1-\epsilon)} / r .
$$

This implies

$$
Q(r) \geq 2 \mu \sqrt{\epsilon(1-\epsilon)} \ln (r)+C
$$

Comparing the upper and lower bounds for $Q(r)$ for large $r$ yields

$$
\beta \geq 2 \mu \sqrt{\epsilon(1-\epsilon)}-\epsilon(n-2)
$$

Since this holds for every $\epsilon \in[0,1]$, the theorem now follows from the computation

$$
\max _{\epsilon \in[0,1]} 2 \mu \sqrt{\epsilon(1-\epsilon)}-\epsilon(n-2)=-\frac{n-2}{2}+\sqrt{\left(\frac{n-2}{2}\right)^{2}+\mu^{2}}=\beta(\mu)
$$

\section{References}

[B] M. T. Barlow, On the Liouville property for divergence form operators, Canad. J. Math. 50 (1998), no. 3, $487-496$.

[BCN] H. Berestycki, L. Caffarelli, L. Nirenberg, Further qualitative properties for elliptic equations in unbounded domains, Ann. Scuola Norm. Sup. Pisa Cl. Sci. (4) 25 (1997), no. 1-2, 69-94 (1998). 
[GG] N. Ghoussoub, C. Gui, On a conjecture of De Giorgi and some related problems, Math. Ann. 311 (1998), no. $3,481-491$.

[L] A. G. Losev, Some Liouville theorems on Riemannian manifolds of a special type, Izv. Vyssh. Uchebn. Zaved. Mat. 1991 no. 12, 15-24; English Transl., Soviet Math. (Iz. VUZ) 35 (1991), no. 12, 15-23.

[LM] A. G. Losev, E. A. Mazepa, Bounded solutions of the Schrödinger equation on Riemannian products, (Russian. Russian summary) Algebra i Analiz 13 (2001), no. 1, 84-110; translation in St. Petersburg Math. J. 13 (2002), no. 1, 57-73 